\title{
DERIVING URBAN MASS CONCENTRATIONS USING TANDEM-X AND SENTINEL-2 DATA FOR THE ASSESSMENT OF MORPHOLOGICAL POLYCENTRICITY
}

\author{
Ines Standfu $\beta^{1}$, Christian Geiß ${ }^{1}$, Marlene Kühnl ${ }^{1}$, Michael Wurm ${ }^{1}$, Stefan Siedentop ${ }^{2}$, Bastian Heider ${ }^{2}$, \\ Hannes Taubenböck ${ }^{l}$
}

\author{
${ }^{1}$ German Remote Sensing Data Center (DFD), German Aerospace Center (DLR), Wessling, Germany \\ ${ }^{2}$ ILS - Research Institute for Regional and Urban Development, Dortmund, Germany
}

\begin{abstract}
Polycentricity refers to urban regions with more than one center. These additional (sub-) centers, e.g. spatial concentrations of jobs, are characteristic for the transformation of monocentric towards polycentric urban patterns. Frequently assessed with socioeconomic data, the phenomenon is also reflected in the built morphology of urban landscapes. Only recently, a methodology for largescale morphological characterization of built-up structures in urban areas relying on TanDEM-X and Sentinel-2 data has been introduced. Thus, a new way to investigate morphologic polycentricity in and among cities is provided. Relying on this approach, we derive the distribution of urban mass concentrations in four city regions. We identify high urban mass concentrations - proxies for (sub-) centers using a threshold approach. A comparison between the studied regions reveals that only one city tends to have a polycentric urban structure. Our study highlights a new and promising possibility to study the urban morphologic development at global scales.
\end{abstract}

Index Terms - urban spatial structure, 3D city structures, urban morphology, TanDEM-X, Sentinel-2

\section{INTRODUCTION}

Cities around the globe are expanding rapidly and undergo constant change [1]. One outcome of the urbanization processes in the Global North has been the transformation of monocentric towards polycentric urban patterns: (sub-) centers have emerged in and around traditional city centers and central business districts [2], [3]. Polycentricity, however, is not a simple concept with an unambiguous definition [4]. It rather comprises a multitude of explanatory approaches and is accordingly difficult to measure.

Often understood as spatial concentration of workplaces [5], the polycentric structure of urban regions is frequently investigated using georeferenced data on socioeconomics like employees or population counts [6]. Though, the potential of such approaches has already reached its limits. Due to data heterogeneity, limited data availability and diversity of strategies for data generation, analyses are restricted to individual regions. An objective comparison between cities or city regions all over the globe relying solely on socioeconomic data is challenging if not impossible [6].

Remote sensing data have the capability to capture objects and patterns of the urban landscape. These morphological characteristics provide an alternative way to measure polycentricity in and among urban areas. A positive relation between the distribution of building volumes and employees in cities has already been pointed out [7]. Given those facts, [6] operationalized (sub-) centers as agglomerations of high urban mass concentrations (hUMC), following [8], [9], and proposed a methodology for (sub-) center detection relying on remote sensing data. The approach combines individual building footprints with a normalized DSM (nDSM) generated from Cartosat-1 data to retrieve building volumes as proposed by [10]. The latter are utilized to retrieve urban mass concentrations (UMC). Those equal the totaled building volume of a reference unit (here a $1 \mathrm{~km}^{2}$ grid) and can be generated for entire city areas and their surroundings. Applying, for instance, a threshold approach then allows for detecting hUMC; a proxy for (sub) centers in the urban landscape.

Although, this represents an improvement compared to the use of heterogenous socioeconomic data, there are still factors that limit the global application of this methodology. High-resolution elevation models, like the one based on Cartosat-1, are expensive and building footprints are not consistently available for every urban region on the planet.

Only recently, an innovative approach to characterize urban morphologies for extended areas has been introduced [11]. It relies on TanDEM-X and Sentinel-2 data to derive built-up volumes of urbanized areas. A clear advantage of this methodology is the utilization of globally available and consistent data sets, making it independent of additional sources like building footprints.

The aim of our study is to characterize the morphologic built-up structure, by means of UMC, within urban environments using the approach proposed by [11]. We do this exemplarily for four city regions, two located in Germany and the United States (US), respectively. Using the UMC as basis, we detect (sub-) centers (hUMC), subsequently, to characterize intra-urban polycentricity 
within the four city regions. Finally, we compare the results to reveal whether the studied regions exhibit rather mono- or polycentric characteristics.

\section{STUDY REGIONS}

Our investigation is focused on the cities of Portland $\left(45^{\circ} 31^{\prime} \mathrm{N}, 122^{\circ} 41^{\prime} \mathrm{W}\right)$ and Atlanta $\left(33^{\circ} 45^{\prime} \mathrm{N}, 84^{\circ} 23^{\prime} \mathrm{W}\right)$ in the US as well as on Hamburg $\left(53^{\circ} 33^{\prime} \mathrm{N}, 10^{\circ} 0^{\prime} \mathrm{E}\right)$ and Hanover $\left(52^{\circ} 22^{\prime} \mathrm{N} 9^{\circ} 43^{\prime} \mathrm{E}\right)$ in Germany. For every city, we uniformly determined a center location using the coordinates representing the geographic midpoints in Open Street Map. Beginning from that center, we included an area of $40 \mathrm{~km}$ radius around each city as a consistent spatial baseline for comparison. These extents ensured that both, administrative areas and parts of the surroundings of the respective cities were considered within our study.

\section{METHOD}

\subsection{Derivation of urban mass concentrations (UMC)}

We used DSM data with a spatial resolution of $12 \mathrm{~m}$ which was generated from TanDEM-X imagery recorded between 2010 and 2015 [12]. Initially, we identified "bare earth" (BE) pixels by applying an iterative region growing-based progressive morphological filter approach [13]. BE pixels are image elements that do not represent objects above ground such as buildings. The initially identified BE pixels are then refined by a joint exploitation of TanDEM-X DSM and Sentinel-2 imagery [14]. Finally, we interpolated the heights of image elements between the identified BE pixel locations using inverse distance weighting. This approach allowed us to generate a DTM with continuous topographic height information. By computing the difference between the original TanDEM-X DSM and the interpolated DTM we obtained a nDSM, containing solely the heights of objects above ground.

To ensure that only the heights of urban objects such as buildings are considered within our investigation, we included another data set; the Global Urban Footprint (GUF) [15]. The GUF is a binary mask discriminating between built-up and non-built up areas with $12 \mathrm{~m}$ spatial resolution and was generated from TerraSAR-X and TanDEM-X data. We spatially intersected the GUF with our nDSM data and retained only built-up pixels with height information.

To retrieve an even more accurate representation of the built-up volume, we excluded above ground objects related to urban vegetation. To do so, we used Sentinel-2 imagery with $10 \mathrm{~m}$ spatial resolution recorded during the autumn/winter periods ( November-March 2010-2015) to calculate a mean NDVI layer. We resampled the nDSM data to fit the $10 \mathrm{~m}$ resolution of the NDVI layer and masked pixels with an NDVI $\geq 0.3$ as vegetation. With these steps done, we generated data sets representing the built-up volume per urban pixel.
In a final step, we spatially combined these built-up volume data with grid layers of $1 \mathrm{~km}^{2}$ cell size to calculate the UMC - the totaled built-up volume - per grid cell. We used the INSPIRE (Infrastructure for Spatial Information in the European Community) grid for Hamburg and Hanover, as proposed by [6], and generated an equal data set for the US cities.

\subsection{Identification of (sub-)centers (hUMC)}

The detection of (sub-)centers was based on the UMC data following the method proposed by [6]. It consists of a combination of regional and distance-based threshold approaches.

In the regional approach, we considered all UMC values of a city region and used a standard deviation (SD) of $>1.3$ as single cut-off value to discriminate hUMC. However, using this approach, the hUMC of the traditional centers or central business districts outshine smaller UMC peaks in the surroundings.

Against this background, a distance-based method is additionally applied. A ring model with $1 \mathrm{~km}$ bandwidth around the center locations of each city is used to assign the UMC grid cells to one of the rings depending on their distance to the center location. For every ring, we defined an individual UMC threshold of $>1.3$ SD to detect hUMC. This enables to detect smaller hUMC in the peripheral areas, too.

In consequence, we combined the results of both approaches and merged neighboring hUMC cells into single (sub-) centers. [6] have empirically determined that this combination and the cut-off value of 1.3 SD facilitate to generate a realistic representation of (sub-) centers.

\subsection{Analysis of morphological polycentricity}

To identify if the considered city regions display rather mono- or polycentric morphological characteristics we derived rank-size distributions as suggested by [6].

We calculated the 2D-area $\left(\mathrm{km}^{2}\right)$ of each detected (sub-) center of the four city regions. Afterwards, we separately sorted the (sub-) centers of every city region by size, with the first rank being assigned to the (sub-) center with the largest 2D-area.

Plotting the rank size distributions of the four cities enables to interpret and compare their individual degree of polycentricity. The main indicator to be considered is the slope - here the difference in area size - between higher and lower ranked (sub-) centers. A steep slope is an indicator for the dominance of a higher ranked (sub-) center which is interpretable as lower degree of polycentricity. A flatter slope may indicate a more balanced distribution of the sizes of (sub-) centers. This can be interpreted as tendency towards a higher degree of polycentric characteristics of a city region. 


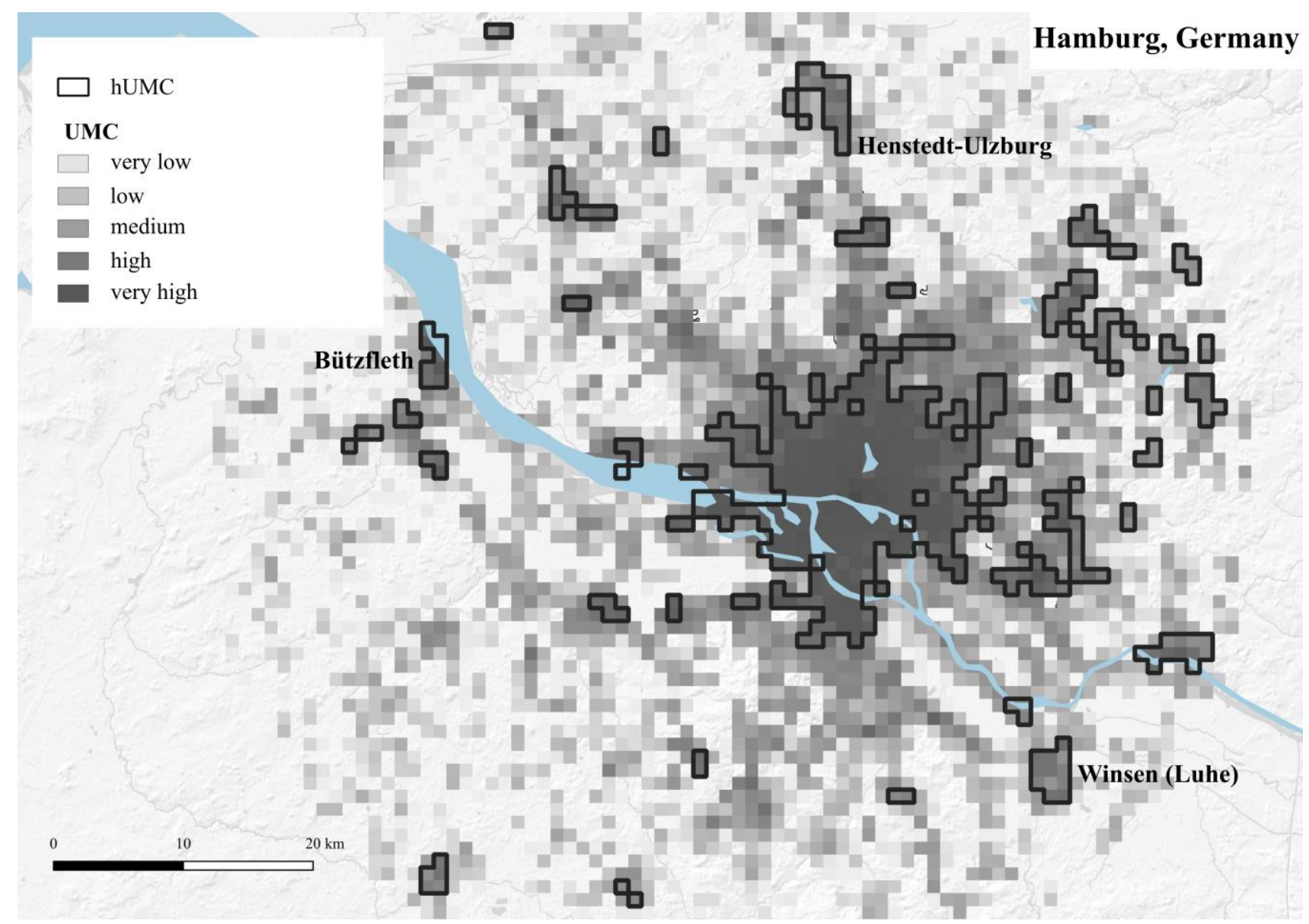

Fig. 1: Distribution of Urban Mass Concentrations (UMC) and detected (sub-) centers (high Urban Mass Concentrations (hUMC)) in Hamburg.

\section{RESULTS}

The generated UMC provide a reasonable representation of the distribution of built-up volumes in our study regions. In Hamburg (Fig. 1), for instance, a large area with high builtup volumes can be spotted in the city center. Those volumes decrease towards the edges of the studied area in the cities' periphery. In addition, communities such as HenstedtUlzberg and Winsen (Luhe) as well as industrial areas like Bützfleth located outside the administrative area of the city, are clearly visible due to an increased concentration of builtup volumes.

The morphological characterization - distribution of UMC - was validated using ordinally weighted overall accuracies and kappa statistics. Although the measured UMC showed high overall accuracies (e.g. $\mathrm{OA}=0.79$ and $\mathrm{k}$ $=0.61$ in Hamburg), a systematic underestimation of built-up heights was uncovered [11]. However, a comparison with official cadastral data revealed that the relative spatial pattern - the distribution of built-up heights - is still well reflected. The influence on the hUMC identification using a relative threshold is therefore assumed to be small or negligible.
Based on the UMC data we identified hUMC - the (sub) centers - in all four city regions. We highlight our results again exemplarily for Hamburg (Fig. 1). In total, we were able to detect 38 (sub-) centers in the considered city region. These comprise a dominant - very large area - city center and some smaller communities in the surroundings of the core city. Remarkedly, the majority of the hUMC in Hamburg have been identified north of the Elbe river.

A comparison of the rank size distributions of the identified (sub-) centers in the four city regions provides an insight on their degree of polycentricity eventually (Fig. 2).

We found a distinctive hierarchy between the identified (sub-) centers in Atlanta, Hamburg and Hanover. The main indicator was the steep slope - strong decrease in size beginning from the centers ranked on position one up to position three. This pattern is presumably resulting from the dominance of the traditional city centers, and thus, an indicator of rather monocentric city characteristics.

The distribution of center sizes in Portland represents a less pronounced hierarchy. Here, the decrease in size between the higher ranked (sub-) centers is way flatter (Fig. 2). 


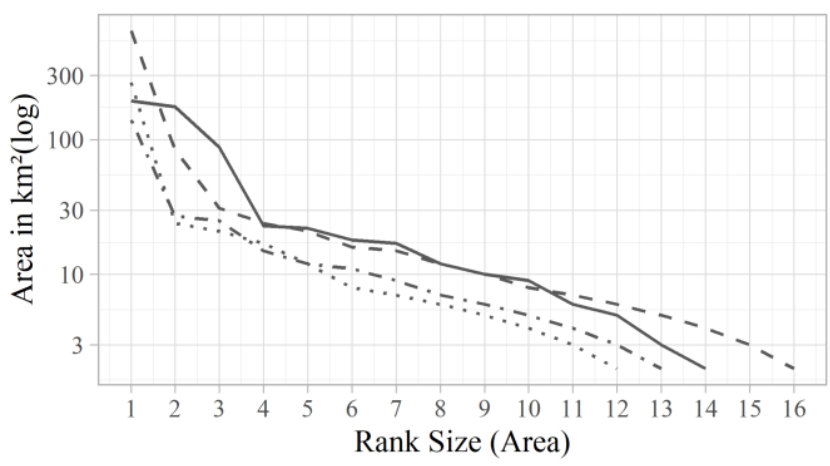

— Portland -- Atlanta -.- Hanover … Hamburg

Fig. 2: Rank size distribution of the detected (sub-) centers in Portland, Atlanta, Hamburg and Hanover.

Therefore, a higher tendency toward polycentric characteristics with less pronounced dominance of the traditional city center is suggested for Portland.

\section{CONCLUSION}

TanDEM-X and Sentinel-2 data allow for extensive and accurate description of built-up structures as proposed by [11]. Thus, a new input data set is provided to identify and study morphological polycentricity. A clear advantage is the use of a globally available and consistent data base that enables not only for national but also intercontinental comparative studies. Although there is potential for further development of this method, it provides an opportunity to reveal comprehensive insights into development paths and effects of planning processes in cities and urban regions around the globe. Moreover, it can contribute to extent current research on the detection of city centers [16].

\section{REFERENCES}

[1] H. Taubenböck et al., "A new ranking of the world's largest cities-Do administrative units obscure morphological realities?," Remote Sens. Environ., vol. 232, no. February, p. 111353, 2019.

[2] A. Anas, R. Arnott, and K. A. Small, "Urban Spatial Structure," J. Econ. Lit., vol. 36, no. 3, pp. 1426-1464, 1998.

[3] D. L. Greene, "Urban Subcenters. Recent Trends in Urban Spatial Structure," Growth Change, vol. 11, no. 1, pp. 2940, 1980.

[4] M. Burger and E. Meijers, "Form follows function? linking morphological and functional polycentricity," Urban Stud., vol. 49, no. 5, pp. 1127-1149, 2012.

[5] D. P. McMillen, "Employment subcenters in Chicago: Past , present, and future," Econ. Perspect., vol. 27, no. 2, pp. 2-14, 2003.

[6] H. Taubenböck, I. Standfuß, M. Wurm, A. Krehl, and S. Siedentop, "Measuring morphological polycentricity - A comparative analysis of urban mass concentrations using remote sensing data," Comput. Environ. Urban Syst., vol. 64, pp. 42-56, 2017.

[7] A. Krehl, "Urban spatial structure: An interaction between employment and built-up volumes," Reg. Stud. Reg. Sci., vol. 2, no. 1, pp. 290-308, 2015.

[8] A. G. Champion, "A changing demographic regime and evolving polycentric urban regions: Consequences for the size, composition and distribution of city populations," Urban Stud., vol. 38, no. 4, pp. 657-677, 2001.

[9] S. Davoudi, "Polycentricity in European spatial planning: From an analytical tool to a normative agenda," Eur. Plan. Stud., vol. 11, no. 8, pp. 979-1000, 2003.

[10] M. Wurm, P. D'Angelo, P. Reinartz, and H. Taubenböck, "Investigating the applicability of Cartosat-1 DEMs and topographic maps to localize large-area urban mass concentrations," IEEE J. Sel. Top. Appl. Earth Obs. Remote Sens., vol. 7, no. 10, pp. 4138-4152, 2014.

[11] C. Geiß et al., "Large-Area Characterization of Urban Morphology-Mapping of Built-Up Height and Density Using TanDEM-X and Sentinel-2 Data," IEEE J. Sel. Top. Appl. Earth Obs. Remote Sens., vol. 12, no. 8, pp. 116, 2019.

[12] B. Wessel, M. Huber, C. Wohlfart, U. Marschalk, D. Kosmann, and A. Roth, "Accuracy assessment of the global TanDEM-X Digital Elevation Model with GPS data," ISPRS J. Photogramm. Remote Sens., vol. 139, pp. 171-182, 2018.

[13] C. Geiß, M. Wurm, M. Breunig, A. Felbier, and H. Taubenböck, "Normalization of TanDEM-X DSM data in urban environments with morphological filters," IEEE Trans. Geosci. Remote Sens., vol. 53, no. 8, pp. 43484362, 2015.

[14] C. Geiß, P. A. Pelizari, S. Bauer, A. Schmitt, and H. Taubenbock, "Automatic Training Set Compilation With Multisource Geodata for DTM Generation From the TanDEM-X DSM," IEEE Geosci. Remote Sens. Lett., pp. $1-5,2019$.

[15] T. Esch et al., "TanDEM-X mission-new perspectives for the inventory and monitoring of global settlement patterns," J. Appl. Remote Sens., vol. 6, no. 1, p. 061702 (2012)., 2012.

[16] M. Wurm, J. Goebel, G. G. Wagner, M. Weigand, S. Dech, and H. Taubenböck, "Inferring floor area ratio thresholds for the delineation of city centers based on cognitive perception," Environ. Plan. B Urban Anal. City Sci., 2019. 